2012;13(4):213-224.

18. Bensinger SJ, Tontonoz P. Integration of metabolism and inflammation by lipid-activated nuclear receptors. Nature. 2008;454(7203):470-477.

19. Zelcer N, Hong C, Boyadjian R, Tontonoz P. LXR regulates cholesterol uptake through Idol-dependent ubiquitination of the LDL receptor. Science. 2009;325(5936):100-104.

20. Rong X, et al. LXRs regulate ER stress and inflammation through dynamic modulation of membrane phospholipid composition. Cell Metab.
2013;18(5):685-697.

21. A-Gonzalez N, et al. Apoptotic cells promote their own clearance and immune tolerance through activation of the nuclear receptor LXR. Immunity. 2009;31(2):245-258.

22. Hong C, et al. Coordinate regulation of neutrophil homeostasis by liver $\mathrm{X}$ receptors in mice. J Clin Invest. 2012;122(1):337-347.

23. Bensinger SJ, et al. LXR signaling couples sterol metabolism to proliferation in the acquired immune response. Cell. 2008;134(1):97-111.
24. Solt LA, Kamenecka TM, Burris TP. LXR-mediated inhibition of $\mathrm{CD}^{+} \mathrm{T}$ helper cells. PLoS One. 2012;7(9):e46615.

25. Gévry N, Schoonjans K, Guay F, Murphy BD. Cholesterol supply and SREBPs modulate transcription of the Niemann-Pick C-1 gene in steroidogenic tissues. J Lipid Res. 2008;49(5):1024-1033.

26. Horton JD, Goldstein JL, Brown MS. SREBPs: activators of the complete program of cholesterol and fatty acid synthesis in the liver. J Clin Invest. 2002;109(9):1125-1131.

\title{
Paradoxical insights into whole body metabolic adaptations following SGLT2 inhibition
}

\author{
William T. Cefalu \\ Pennington Biomedical Research Center, Baton Rouge, Louisiana, USA.
}

\begin{abstract}
It is well known that glycemic control over time reduces microvascular and macrovascular complications in human subjects with type 2 diabetes. In addition, preclinical models of type 2 diabetes have demonstrated that long-term hyperglycemia exacerbates insulin resistance and reduces $\beta$ cell function; therefore, therapies that reduce blood glucose levels are of great interest in not only controlling complications, but for restoring known defects in the pathogenesis of type 2 diabetes. Pharmacological inhibition of the sodiumglucose cotransporter 2 (SGLT2) reduces plasma glucose by limiting glucose absorption in the kidney and increasing glucose excretion in the urine. In this issue of the JCI, Merovci and colleagues and Ferrannini and colleagues independently report a paradoxical increase in endogenous glucose production in patients with type 2 diabetes following SGLT2 inhibition, despite an overall decrease in fasting plasma glucose. Together, these studies provide a unique insight into the effects of SGLT2 inhibition on whole body metabolism.
\end{abstract}

\section{Pharmacological control of hyperglycemia}

The natural history of type 2 diabetes (T2D) is characterized by a progressive decline in insulin secretion, development of insulin resistance, and increased hepatic glucose production. Based on these observations, it is generally accepted that after an initial period of lifestyle management and monotherapy (usually metformin), a combination of therapies designed to address T2D-associated pathophysiology will be required to effectively manage hyperglycemia over time (1). Currently, clinicians can chose from a large number of agents (e.g.,

Conflict of interest: William T. Cefalu has served as a consultant for Intarcia Therapeutics Inc. and, on behalf of his institution, has consulted for Shire, Novo Nordisk, and Sanofi-Aventis. He has served as principal investigator on research studies with funding awarded to his institution from AstraZeneca, Bristol-Myers Squibb, Sanofi-Aventis, Johnson \& Johnson, MannKind Corporation, GlaxoSmithKline, and Lexicon Pharmaceuticals Inc.

Citation for this article: J Clin Invest. 2014; 124(2):485-487. doi:10.1172/JCI74297. sulfonylureas, thiazolidinediones, incretin therapies, and exogenous insulin) that each have a unique mechanism of action, which makes each an appropriate add-on to the metformin background therapy (1). Although all of these agents are considered to provide efficacy on glycemic control, many of them produce adverse side effects that ultimately dictate their use. Thus, there is clearly a need for pharmacotherapy that can improve glycemia yet have a beneficial effect on unmet clinical needs, including weight gain and hypoglycemia. In this regard, there has been great interest in the sodium-glucose cotransporter 2 (SGLT2), because pharmacologic inhibition of this glucose cotransporter offers an attractive approach to modulate carbohydrate metabolism (2). Specifically, prior studies on SGLT2 inhibitors revealed that these agents lower the renal threshold for glucose and reduce glucose absorption in the kidney, resulting in increased urinary glucose excretion, decreased plasma glucose and glycated hemoglobin (A1c) lev- els, mild osmotic diuresis, and a favorable effect on weight (2-5). In essence, SGLT2 inhibition and associated net reduction in renal glucose reabsorption have provided a new insulin-independent approach for treatment of T2D.

To date, there are a number of SGLT2 inhibitors available currently or in development. Despite substantial clinical data on the effects of these inhibitors on glycemic control and other clinical parameters, there is a paucity of data in humans that comprehensively evaluates the whole body metabolic adaptation to pharmacologic inhibition of the renal SGLT2 cotransporter. In this issue of the JCI, results are presented from two elegantly conducted metabolic studies in individuals with T2D that evaluated the SGLT2 inhibitors, dapagliflozin and empagliflozin $(6,7)$. Both studies were well designed, used sophisticated metabolic techniques, evaluated subjects with T2D, involved both acute and longer-term evaluation (two- and four-week observations), and evaluated whole body insulin sensitivity and endogenous hepatic glucose production in response to SGLT2 inhibition. Although it appears that the primary aims and study approaches differed, it is interesting to note that both trials confirmed a surprising finding: SGLT2 inhibition elicits a paradoxical rise in endogenous glucose production (EGP), despite lowering fasting glucose.

\section{Testing the glucotoxicity hypothesis} in patients with T2D

In the study by Merovci et al. (6), the primary goal was to evaluate the impact of hyperglycemia and its reduction by SGLT2 
inhibition on whole body insulin action. In preclinical models, hyperglycemia exacerbates two core defects of T2D, insulin resistance and decreased $\beta$ cell function (8); however, this "glucotoxicity hypothesis" has not been adequately demonstrated in humans, given the possible confounders associated with the method of glucose lowering used in prior studies. Thus, Merovci and colleagues set out to determine whether the glucotoxicity hypothesis was operative in humans and provided a welldescribed and justified rationale for using a SGLT2 inhibitor to lower plasma glucose concentration and assessed its effect on insulin action, without altering other metabolic processes. Using the hyperinsulinemic euglycemic clamp technique, which is the gold standard for assessing insulin action, Merovci et al. clearly demonstrated that the SGLT2 inhibitor dapagliflozin induces glycosuria, lowers fasting plasma glucose, and substantially increases insulin action after two weeks compared with placebo. After accounting for urinary glucose loss, whole body glucose disposal remained significantly elevated in patients taking the SGLT2 inhibitor. The demonstration that the insulin action impairment in hyperglycemic patients can be reversed by improvements in glycemia, as a result of dapagliflozin-induced glucosuria, supports operation of the glucotoxicity hypothesis in humans. Interestingly, Merovci and colleagues evaluated EGP following dapagliflozin or placebo and paradoxically reported that EGP actually increases with SGLT2 inhibition.

\section{Evaluation of SGLT2 inhibitors in patients with T2D}

In a related study in this issue of the JCI, Ferrannini et al. (7) evaluated subjects with T2D at baseline and after administration of the SGLT2 inhibitor empagliflozin. Patients were evaluated following their first dose of empagliflozin $(25 \mathrm{mg})$ and after extended empagliflozin administration $(25 \mathrm{mg} / \mathrm{d}$ for 28 days). At both time points, Ferrannini and colleagues used a mixed meal with a double-tracer technique to assess meal-derived glucose, EGP, and insulin sensitivity. Patients exhibited the expected glycosuria following empagliflozin treatment. In agreement with Merovci et al. (6), Ferrannini and colleagues also found that SGLT2 inhibition (in this case with empagliflozin) increases EGP. Furthermore, Ferrannini et al. demonstrated an increase in $\beta$ cell glucose sen- sitivity and an estimated insulin sensitivity that was significantly increased during the acute study and "was at least maintained as compared to baseline" in the chronic study (7). Ferrannini and colleagues provided much more detail regarding the components of total glucose disposal, as they also assessed substrate utilization. The results of their chronic study may be of great clinical interest, as these patients exhibited a larger decrement in tissue glucose disposal, which was reflected not only by a decrease in nonoxidative glucose disposal, but by decreased glucose oxidation as well. Lipid oxidation was increased in these patients to match the decrease in glucose oxidation, thereby maintaining energy balance. Chronic treatment with the SGLT2 inhibitor clearly appeared to shift substrate utilization from carbohydrate to lipids. The combined observations that inhibition of SGLT2 did not result in a reduced rate of energy expenditure and that weight loss in clinical trials has been less than predicted provide support for the suggestion made by Ferrannini et al. that the agent may potentially increase dietary intake. This is an intriguing hypothesis; however, at this time, it will need to be specifically validated with techniques and procedures that can accurately assess the effects of SGLT2 inhibition on lipid and glucose utilization.

\section{The paradox}

Strikingly, both the study by Merovci et al. and the study by Ferrannini et al. revealed that despite a clinical reduction in fasting hyperglycemia following SGLT2 inhibition, EGP apparently increased with use of these agents. Given that fasting glucose was significantly lowered with dapagliflozin and empagliflozin, and given the close relationship between EGP and fasting plasma glucose, it would be expected that SGLT2 inhibition would decrease EGP (9). The observation that neither dapagliflozin nor empagliflozin reduced EGP despite the lowering of blood glucose is clearly a surprising finding that would be considered a paradoxical response. Given the careful attention to study design and use of the precise metabolic techniques, this finding is clearly supported and confirmed by the results of both Merovici and colleagues and Ferrannini and colleagues. The fact that each study evaluated a different SGLT2 inhibitor indicates that increased EGP can be ascribed to SGLT2 inhibition per se and representative of the SGLT2 inhibitor class, rather than to a specific effect of any particular agent.
Differences were reported on the magnitude of EGP increases between the studies and for adequate compensation. For example, Merovci et al. (6) suggested that the increase in EGP occurring on a daily basis may not offset the increase in daily glucose excretion. Specifically, Merovci and colleagues reported that the amount of glucose appearing in the circulation on day 3 was approximately half of the amount of glucose observed in the urine following SGLT2 inhibition. On the other hand, Ferrannini et al. (7) suggested that the rise in EGP associated with a single dose of SGLT2 inhibitor exactly balanced the glucose lost through the urine over a three-hour period. Both studies investigated possible contributing mechanisms and suggested that SGLT2 inhibition promotes an increase in plasma glucagon, a decrease in plasma insulin, and an increase in the glucagon/insulin ratio, resulting in increased hepatic glucose production.

\section{Relevance and future directions}

What do the current studies by Ferrannini et al. and Merovci et al. contribute to the literature and clinical medicine? First and foremost, these studies provide a better appreciation of the independent effects of glucose on peripheral insulin action. Interestingly, the classic preclinical study on glucotoxicity and insulin action that served as a rationale for the current human evaluation was reported in the JCI over 25 years ago by members of the Merovci et al. group (10). It is clear that in addition to long-term reduction in diabetic complications from glycemic control, acute correction of hyperglycemia has substantial metabolic effects. Second, the information that SGLT2 inhibition increases EGP despite reducing fasting glucose is interesting not only from a physiologic perspective, which considers metabolic response to enhanced glycosuria, but also from a clinical perspective. Should the mechanism underlying the SGLT2 inhibition-associated increase in EGP be validated, Merovci et al. proposed an interesting approach to clinical treatment (6). Specifically, Merovci and colleagues suggest that a combination of SGLT2 inhibition with incretin therapies, which are anticipated to attenuate the increase in EGP, would provide synergism for treatment of hyperglycemia.

In summary, the studies by Merovci et al. and Ferrannini et al. are examples of classic, in-depth human metabolic research and provide important contributions to 
the field. Together, these studies provide new information on whole body metabolic adaptations to SGLT2 inhibition and lay the groundwork for additional research, particularly relating to the paradoxical rise in EGP. Some questions are still left unanswered. Would a combination of SGLT2 inhibitors with agents such as incretins address the increase in EGP? Does the discrepancy between calories lost in the urine and total weight loss really indicate that SGLT2 inhibitors increase caloric intake? Clearly, additional metabolic studies are needed and should provide additional clarification.

\section{Acknowledgments}

W.T. Cefalu is supported in part by grant 1 U54 GM104940 from the National Institute of General Medical Sciences of the National Institutes of Health, which funds the Louisiana Clinical and Translational Science Center; by grant P50AT002776 from the National Center for Complementary and Alternative Medicine (NCCAM) and the Office of Dietary Supplements (ODS), which funds the Botanical Research Center; and from NIH grant P30DK074276, which funds the Nutrition Obesity Research Center.

Address correspondence to: William T. Cefalu, Douglas L. Manship, Sr. Professor Diabetes, Executive Director, Pennington Biomedical Research Center, 6400 Perkins Road, Baton Rouge, Louisiana 70808, USA. Phone: 225.763.2654; Fax: 225.763.3030; E-mail: cefaluwt@pbrc.edu.

1. Inzucchi SE, et al. Management of hyperglycemia in type 2 diabetes: a patient-centered approach. Position statement of the American Diabetes Association (ADA) and the European Association for the Study of Diabetes (EASD). Diabetes Care. 2012;35(6):1364-1379.

2. DeFronzo RA, Davidson JA, Del Prato S. The role of the kidneys in glucose homeostasis: a new path towards normalizing glycaemia. Diabetes Obes Metab. 2012;14(1):5-14

3. Monami M, Nardini C, Mannucci E. Efficacy and safety of sodium glucose co-transport-2 inhibitors in type 2 diabetes: a meta-analysis of randomized clinical trials [published online ahead of print December 5, 2013]. Diabetes Obes Metab. doi:10.1111/dom.12244.

4. Cefalu WT, et al. Efficacy and safety of canagliflozin versus glimepiride in patients with type 2 diabetes inadequately controlled with metformin (CANTATA-SU): 52 week results from a randomised, double-blind, phase 3 non-inferiority trial. Lancet. 2013;382(9896):941-950.

5. Goring SM, et al. Dapagliflozin compared with other oral anti-diabetes treatments when added to metformin monotherapy: a systematic review and network meta-analysis [published online ahead of print November 14, 2013]. Diabetes Obes Metab. doi:10.1111/dom.12239.

6. Merovci A, et al. Dapagliflozin improves muscle insulin sensitivity but enhances endogenous glucose production. J Clin Invest. 2014;124(2):509-514.

7. Ferrannini E, et al. Metabolic response to sodiumglucose cotransporter 2 inhibition in type 2 diabetic patients. J Clin Invest. 2014;124(2):499-508.

8. Rossetti L, Giaccari A, DeFronzo RA. Glucose toxicity. Diabetes Care. 1990;13(6):610-630.

9. DeFronzo RA, Ferrannini E, Simonson DC. Fasting hyperglycemia in non-insulin-dependent diabetes mellitus: contributions of excessive hepatic glucose production and impaired tissue glucose uptake. Metabolism. 1989;38(4):387-395.

10. Rossetti L, Smith D, Shulman GI, Papachristou D, DeFronzo RA. Correction of hyperglycemia with phlorizin normalizes tissue sensitivity to insulin in diabetic rats. J Clin Invest. 1987;79(5):1510-1515.

\title{
SMN-targeted therapeutics for spinal muscular atrophy: are we SMArt enough yet?
}

\author{
Kathryn J. Swoboda
}

Department of Neurology, University of Utah School of Medicine, Salt Lake City, Utah, USA.

\begin{abstract}
Spinal muscular atrophy (SMA) remains one of the most common and lethal autosomal recessive diseases. Homozygous deletion of survival of motor neuron 1 (SMN1) and resulting SMN protein deficiency manifests predominantly with motor neuron degeneration; however, a wealth of emerging data supports a broader influence of SMN deficiency in disease pathogenesis. In this issue of the JCI, Kariya and colleagues demonstrate the relatively selective impact of SMN depletion on the distal motor unit using a series of SMN2-expressing transgenic mice in which constitutive SMN knockdown follows variable periods of normal development. Their observations provide further insights regarding the temporal requirements for SMN in mice, renewing speculation about when and where repletion of SMN is necessary for optimal outcomes in SMA patients.
\end{abstract}

\section{SMN-targeted therapeutics: the hope and the hype}

Spinal muscular atrophy (SMA) is an autosomal recessive disorder characterized by motor neuron loss in the spinal

Conflict of interest: Kathryn J. Swoboda has clinical trial contracts with ISIS Pharmaceuticals and Roche Pharmaceuticals for clinical studies or trials in SMA patients.

Citation for this article: J Clin Invest. 2014; 124(2):487-490. doi:10.1172/JCI74142. cord and brainstem. Progressive muscular weakness and atrophy typically emerge in infancy or early childhood after a variable period of normal development. Deficiency in survival motoneuron ( $\mathrm{SMN}$ ) is associated with SMA. In humans and bonobos, a unique inverted duplication on chromosome 5 encompasses SMN1 and its nearly identical homolog, SMN2; however, lower vertebrates, including mice, lack SMN2. Deletion and/or mutation of SMN1 is dis- ease causing, and a translationally silent nucleotide substitution in SMN2 results in a truncated, less stable protein. Historically, low SMN was thought to selectively target motor neurons; however, emerging data from SMA mouse models indicate that low levels of SMN affect many tissues, including components of the neuromuscular circuitry, and skeletal muscle (1). In addition to obvious defects in neuromuscular junction (NMJ) maturation, decreased SMN results in early abnormalities in synaptic input from muscle fibers to motor neurons within the spinal cord $(2,3)$. Both SMA mouse models and severely affected human infants exhibit abnormalities in myofiber maturation, muscle size, and muscle function (4-6). Furthermore, there is evidence that $\mathrm{SMN}$-intrinsic defects in Schwann cells promote SMA pathogenesis, and peripheral nerve myelination is abnormal in severely affected infants (7). In humans lacking SMN production from SMN1, SMN2 phenotypically modifies 